\title{
Una mirada al abandono escolar prematuro en Portugal: realidades, contextos y prácticas
}

\section{A look at early school leaving in Portugal: Realities, contexts and practices}

\author{
Helena Costa Araújo*1 \\ haraujo@fpce.up.pt \\ EUNiCE MACEDO* \\ eunice@fpce.up.pt \\ António Magalhães** \\ antonio@fpce.up.pt \\ Cristina Rocha* \\ crocha@fpce.up.pt \\ *Centro de Investigação e Intervenção Educativas, Porto, Portugal \\ **Centro de Investigação de Políticas de Ensino Superior, Porto, Portugal
}

\section{Resumen:}

Teniendo en cuenta que el abandono prematuro de la escuela (ESL)/ abandono prematuro de la educación y de la formación es un problema sumamente político, este articulo hace una mirada al abandono escolar prematuro en Portugal, enfocando sus realidades, contextos y prácticas, en una interacción entre el nivel nacional y europeo y sus orientaciones educativas. Se hace un breve análisis del contexto del problema y su evolución y se presenta el sistema educativo para después reflexionar acerca de la actual orientación educativa, aportando evidencias sobre las perspectivas de inclusión. Finalmente se enfocan medidas intramuros para enfrentar el abandono escolar en una escuela secundaria superior en un área de intervención educativa prioritaria. El objetivo del

\begin{abstract}
:
Bearing in mind that early school leaving (ESL) / early leaving from education and training (ELET) is a political problem, this article looks at early school leaving in Portugal, focusing on its realities, contexts and practices and tapping into the interaction between the national and the European levels and their guidelines for education. The context of the problem and its evolution is briefly analyzed. Following the description of the education system, we also provide some reflections on current educational lines, providing evidence on how inclusion is implemented within such lines. Finally, intramural measures are proposed with a view to addressing early school leaving in an upper secondary school located in an educational area of priority intervention. The objective of the
\end{abstract}

1 Dirección para correspondencia (correspondence address):

María Teresa González González. Universidad de Murcia. Departamento de Didáctica y Organización Escolar. Facultad de Educación. Campus de Espinardo, s/n. 30100 Murcia (España). 
trabajo es analizar el abandono prematuro de la escuela, hacendo oír las voces de diferentes personas implicadas, como son el diretor de la escuela, el profesorado y las/ los jóvenes ante un conjunto de medidas implementadas en su contexto educativo. Estas medidas son preventivas, e incluyen una preparación para los exámenes y el defensor del estudiante, cuestiones centrales en este artículo. Se argumenta que la lucha contra ESL se encuentra en la tensión entre las políticas destinadas a hacer frente a las necesidades de la economía y de la sociedad del conocimiento y la tentativa de promover la inclusión social a través de la consecución de los itinerarios educativos, dando herramientas a las personas jóvenes para llevar adelante sus posibles opciones.

\section{Palabras clave:}

Abandono escolar prematuro; jóvenes; políticas educativas; medidas educativas article is to analyze early school leaving by making the voices of different stakeholders heard-the school principal, teachers and students-following the implementation of a set of educational measures. These measures are preventive and include preparation for exams and the function of student advocates, two aspects which have a prominent role in this article. We argue that the struggle against ESL lies in the tension between economic policies and the knowledge society and the promotion of social inclusion which requires the definition of educational trajectories aimed at providing young people with the necessary tools for them to make adequate decisions for their future.

\section{Key words:}

Early school leaving; young people; education policies; education measures

\title{
Résumé:
}

Sachant que le décrochage scolaire est un problème politique extrême, cet article examine le décrochage scolaire au Portugal, en se concentrant sur ses réalités, contextes et pratiques. II reconnaît l'interaction entre le niveau national et européen et ses lignes directrices pour l'éducation. Une brève analyse des contours du problème et de son évolution est faite et nous présentons le système éducatif. L'article revient ensuite sur les orientations éducatives actuelles, témoignant des perspectives d'inclusion. Enfin, nous parlons des mesures intra-muros qui visent à lutter contre le décrochage scolaire précoce d'un lycée dans une zone d'intervention éducative prioritaire. L'objectif de l'article est d'analyser le décrochage scolaire en faisant entendre les voix des différentes parties prenantes - le directeur de l'école, les enseignants et les jeunes - face à un ensemble de mesures mises en œuvre dans leur contexte éducatif. Les mesures sont préventives et comprennent la préparation aux examens et le défenseur des élèves, sur lesquelles I'accent est mis dans cet article. Nous soutenons que la lutte contre le décrochage scolaire précoce réside dans la tension entre les politiques visant à répondre aux besoins de l'économie et de la société de la connaissance et la tentative de promouvoir l'inclusion sociale par la réalisation de trajectoires éducatives, fournissant aux jeunes des outils pour réaliser leurs possibles options.

\section{Mots clés:}

Décrochage scolaire précoce ; jeunes ; politiques éducatives; mesures éducatives

\author{
Fecha de recepción: 13-02-2020 \\ Fecha de aceptación: 15-03-2020
}




\section{Introducion}

En este artículo se analizará cómo el problema del abandono prematuro de la escuela (ESL) ha sido tratado en Portugal desde el año 2015. Se parte de un enfoque a nivel macro, se aborda un nivel meso y por último un nivel micro. Se busca, de un lado, entender cómo el país se ha enfrentado a la preocupación europea por el ESL a través de políticas y estudios que buscan reflexionar sobre las medidas para compara combatirlo. Por otra parte, este artículo se centra en medidas intramuros en escuelas secundarias, tomadas con el fin de incorporar medidas y prácticas que enfrentan los procesos de adandono escolar prematuro a nivel local. El ejemplo de dos escoles secundarias que participan en el proyecto RESL. eu puede ser relevante en la comprensión de los propósitos de las medidas adoptadas.

El artículo se basa en un proyecto que involucró a nueve países europeos y utilizó una metodología mixta cuantitativa y cualitativa, respaldada por encuestas a gran escala, entrevistas y discusiones grupales enfocadas. Este artículo utiliza datos recopilados a través de entrevistas individuales con el director y discusión grupal enfocada, realizada tanto con maestros/as como con estudiantes.

El abandono escolar prematuro (Early School Leaving - ESL) ha sido identificado en la Unión Europea como un problema social grave, con diferentes contornos nacionales y locales. Tiene consecuencias preocupantes para el individuo y para el desarrollo social y económico (OECD, 2013, 2018). De acuerdo con Eurostat "ESL es un indicador que identifica la tasa de la población de 18 a 24 años que ha concluido la educación secundaria inferior [ $3^{\circ}$ ciclo de la enseñanza básica] y no está estudiando o asistiendo a ningún tipo de formación profesional". El Consejo Nacional de Educación (CNE), órgano asesor independiente del Departamento de Estado de Educación, es una de las instituciones que aclaran ESL en su glosario y en sus textos. El CNE sigue la definición de Eurostat (CNE, 2010, 2011, 2012). Con el fin de poder establecer relaciones entre países cuyos sistemas educativos son muy diferentes, el proyecto RESL. eu ha creado un concepto de manejo de abandono escolar prematuro que viene asociado solamente a la salida de la escuela sin concluir el $12^{\mathrm{a}}$ grado o equivalente (ISCED 3).

La integración europea en la múltiple escala se configura más como un sistema político que como un proceso legal (Borrás y Jacobsson, 
2004). Entonces, el análisis de la interacción entre los niveles nacional y europeo debe permitir explorar la dialéctica de discursos entre el nivel nacional y el nivel europeo (Dale, 2007). Asi, los distintos gobiernos nacionales han utilizado las oportunidades y limitaciones de esta interacción, para impulsar políticas dirigidas a la integración europea y para implementar políticas nacionales a través de políticas de renacionalización (Musselin y Paradeise, 2009). A su vez, la Estrategia Europa 2020 da prioridad a que la economía europea sea más sostenible, inclusiva e inteligente; y desafía a los Estados para proporcionar empleo, productividad y cohesión social. La reducción de las tasas de abandono escolar al $10 \%$ es uno de los objetivos principales de la UE (Comissão Europeia, 2010).

Sin embargo, el tratamiento estadístico de ESL (Eurostat, 2019), haciendo su make up estadístico, puede ocultar problemas de desigualdad social (Tarabini y Montes, 2015) y de potencial segregación educativa asociada a vías educativas a las que asigna diferentes valores sociales. Por esto, se justifica considerar el ESL un problema sumamente político.

En términos estadísticos, Portugal sigue teniendo tasas altas de ESL en comparación con otros países europeos y con la media europea del 13\% (Autor1, 2014). A pesar de eso, el porcentaje de abandono escolar prematuro ha disminuído visiblemente en las últimas décadas, del $47 \%$ en 1993 al 19\% en 2013, conforme datos de Pordata, en 2014². La tasa de abandono prematuro de la educación y de la formación hizo hincapié en la mejora en 2014, cayendo a 17,4\%. Ya en 2015, el ELET (Early Leaving from Education and Training) cayó al 13,7\%, conforme dados de Eurostat, en 201633. Cabe señalar que el indicador ELET sustituyó al "abandono escolar prematuro", centrado en la educación, para incorporar el abandono de otros espacios de aprendizaje y de formación fuera de la escuela, que también proporcionan un grado. Dada la variada oferta educativa para la finalización de la educación secundaria y la extensión de la enseñanza obligatoria hasta los 18 años (o la finalización del año 12, o equivalente a la ISCED 3 - International Standard Classification of Education), Portugal está más cerca de la meta europea. Si "en 2018, un promedio del 10,6\% de jóvenes (de 18 a 24 años) en la UE aban-

2 http://www.pordata.pt/Europa/Taxa+de+abandono+precoce+de+educa\%C3\%A7\%C $3 \% \mathrm{~A} 3 \mathrm{O}+\mathrm{e}+$ forma $\% \mathrm{C} 3 \% \mathrm{~A} 7 \% \mathrm{C} 3 \% \mathrm{~A} 3 \mathrm{o}+$ total + e+por+sexo- 1350

3 Early leavers from education and training (total), retirado em 01-04-2016 http://appsso. eurostat.ec.europa.eu/nui/show.do?dataset=edat_Ifse_14\&lang=en 
donaron la educación y la formación (...) las mayores reducciones (en términos de puntos porcentuales) entre 2013 y 2018 en la proporción de abandonos prematuros se produjeron en Portugal, España y Grecia, cada una con una caída superior a 5.0 puntos"; en Portugal el el valor actual es $11.8 \%$ (Eurostat, 2019, para 3-5).

A principios del siglo XXI, en el contexto portugués, en conjunción con los retos y las directrices europeas, donde se cruza la inversión en la educación y la integración de las y los jóvenes en el mercado laboral, el enfoque político en el ESL se ha situado especialmente en la tensión entre la inversión en medidas para la competitividad en el mercado laboral y la búsqueda de la igualdad y la inclusión educativa y social (Autor2, 2015; Autor3, 2015). Como se muestra delante, en diferentes períodos, estos brotes se articulan con las políticas gubernamentales de los partidos en el gobierno, que no siempre han tomado la ESL como una prioridad y no se enfrentó a él consecuentemente. Esto también se debe a la aplicación de medidas para reformar el sistema con una tendencia por cada gobierno a sustituir a las políticas existentes por otros que se consideren más ajustados sin que la aplicación de las políticas anteriores hubiera sido evaluada (Silva, 2012).

El sistema educativo portugués sigue siendo bastante centralizado incluso en lo que respecta a las escuelas con mayor autonomía como es el caso de las dos escuelas mencionadas a continuación. Esta estrategia política ha demostrado no ser operativa. La reivindicación de una política nacional más eficaz sobre el ESL emerge en las entrevistas, bajo el proyecto RESL.eu, del número total de encuestados de la encuesta con profesores de la secundaria que trabajan en algunas escuelas del país. Esto parece expresar una preocupación que es común en la profesión docente (Stoleroff y Pereira, 2009). Otra cuestión que preocupa al profesorado, y que afecta a las escuelas públicas, es la organización en grandes conglomerados escolares que hacen complicada la gestión y el funcionamiento de las escuelas involucradas (Autor1, 2015).

El monitoreo de ESL es otro problema que todavía se enfrenta al país, ya que no hay suficientes datos. Cuando un/a estudiante deja una escuela es difícil darse cuenta si se convirtió en un ESLer (jóvenes de 18 a 24 años que abandonaron la escuela sin terminar el grado 12) o simplemente se trasladó a otra escuela o hizo una breve interrupción en sus estudios. Sin embargo, la colaboración con los empleadores y otras agencias 
externas también fue identificada por el número total de maestros en la encuesta/investigación como una herramienta esencial para tratar con el ESL (Autor1, 2015).

Con el fin de comprender mejor el problema en estudio, presentamos abajo un esquema de la organización del sistema educativo, en Portugal, que relaciona los niveles de escolaridad con las edades de frecuencia.

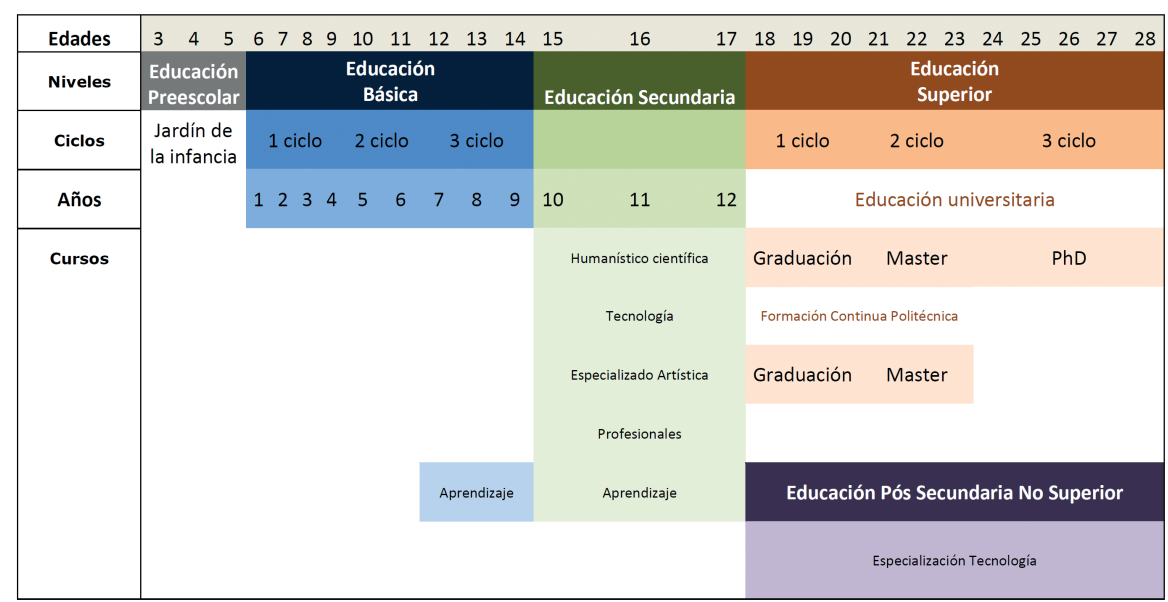

Figura 1. Organización del Sistema Educativo Portugués. ${ }^{4}$

Nota: Los cursos de aprendizaje son de la responsabilitad del Instituto de Empleo y Formación Profesional.

Como ya se ha indicado, la educación es obligatoria hasta el grado 12 o los 18 años de edad. El sistema educativo portugués se organiza en niveles: educación preescolar, la educación básica, educación secundaria y educación superior. Tan solo la educación básica y secundaria es obligatoria. La frecuencia de la educación preescolar es opcional y está destinado a niños de edades comprendidas entre los 3 y los 6 años, la edad de ingreso en el 1 er ciclo de la educación básica. En los últimos años se ha producido la universalización de la educación preescolar para los niños/as de 5 años de edad, preparatoria y promotora del éxito académico en el 1er ciclo. La educación escolar se desarrolla en tres niveles: educación básica - tiene tres ciclos, el $1^{\circ}$ cuatro años, el $2^{\circ}$ dos y el $3^{\circ}$ tres. La educación secundaria - comprende un ciclo de tres

4 Adaptado de Direção Geral da Educação http://euroguidance.gov.pt/index. php?c=int\&id=2, consultado en 08.09.2016. 
años $\left(10^{\circ}, 11^{\circ}\right.$ y $12^{\circ}$ años de estudio). La educación superior incluye la enseñanza universitaria y politécnica. En la enseñanza secundaria, los cursos científicos y humanísticos confieren un diploma para este nivel de educación, orientandose a la búsqueda de los estudios de educación superior. Los cursos de tecnología, especialización artística y profesionales proporcionan un diploma de finalización de la educación secundaria y el nivel de cualificación profesional 3 (Quadro Nacional de Qualificações - Marco Nacional de Cualificaciones) y estan dirigidos para la inserción en el mundo laboral y la continuación de estudios en cursos de post-secundarias de especialización tecnológica o enseñanza superior. Los últimos se caracterizan por el enfoque en habilidades para una profesión, en conjunto con el sector empresarial local. Ya los cursos de aprendizaje proveen un nivel de certificación profesional 2, 3 o 4 y dan la equivalencia al $9^{\circ}$ o $12^{\circ}$ grado. Con una duración media de tres años preparan los jóvenes para el ejercicio de profesiones tituladas, facilitando la entrada en el mercado de trabajo y la continuación de los estudios. En función de su naturaleza y de su público objetivo, estos cursos son responsabilidad del Ministerio de Educación y / o del Instituto de Empleo y Formación Profesional y se enseñan en diferentes instituciones vinculadas o no al Estado ${ }^{5}$.

\section{Orientación política educativa actual en Portugal}

A partir de 2015, con la presencia de un gobierno socialista apoyado por los partidos más a la izquierda, se hace hincapié en la atención a la igualdad y la inclusión, que parecen estar ligadas al refuerzo de los procesos de aprendizaje y desarrollo individual. En este nuevo horizonte de configuración de las opciones políticas debe referirse: la erradicación de las pruebas nacionales en el $4^{\circ}$ y $6^{\circ}$ año (el año de pasaje del 1 er y de los $2^{\circ}$ ciclos de la educación básica) y su sustitución por pruebas de evaluación de los conocimientos adquiridos en los años intermedios para permitir la adopción de medidas compensatorias a finales de estos ciclos; demanda de condiciones para la universalización efectiva de la educación infantil; suministro de libros de texto en el 1er ciclo; y la creación de la figura del profesor-tutor. También es de destacar el orden

5 http://cdp.portodigital.pt/educacao-e-formacao/ensino-basico-e-secundario/modalidades-de-ensino/cursos-cientifico-humanisticos 
político para fomentar la asistencia a la educación profesional, por un lado, reduciendo la naturaleza de estigmatización de estos cursos que ha sido asociada a su desvalorización social tanto como cursos como por las oportunidades profesionales que permiten, y por el otro, haciéndolos más atractivos para las/os estudiantes.

En este período más reciente, hay que enfatizar la Estrategia de Educación Ciudadana (Orden $N^{\circ}$ 6173/2016) que se basa en la intención política de desarrollar el área de Ciudadanía, Derechos Humanos e Igualdad de Género como prioridad nacional y para las y los niños y jóvenes en particular. Se espera que las y los niños y jóvenes practiquen y adquieran habilidades y conocimientos de ciudadanía a lo largo de los diferentes ciclos en términos de valores y conceptos de ciudadanía nacional, derechos humanos, igualdad de género, no discriminación, interculturalidad, inclusión de personas con discapacidad, educación sanitaria, educación en derechos sexuales y reproductivos y educación vial. Los modos de implementación son flexibles y se articulan con las realidades de las escuelas.

A su vez, en 2017, se define también la posibilidad de que las escuelas se unan al proyecto de autonomía y flexibilidad curricular (Orden N - 5908/2017; Decreto-Ley n 55/2018), bajo la experiencia pedagógica, que se implementará más adelante en un nivel más global. La orden establece los principios rectores y las reglas para la concepción, operacionalización y evaluación del currículo de educación primaria y secundaria. La innovación en la organización y el desarrollo curricular como herramienta para promover el éxito y reducir el abandono escolar prematuro, el enfoque en las y los estudiantes y en los aprendizajes esenciales, participación, transdisciplinariedad, autonomía y responsabilidad personal y colectiva son pilares centrales en este proceso.

También prestamos especial atención al establecimiento de los principios y estándares que garantizan la inclusión (Decreto-Ley n 54/2018), buscando responder a la diversidad de necesidades y potencialidades de cada estudiante. Una mayor participación en los procesos de aprendizaje y en la vida de la comunidad educativa, la movilización de las diferentes áreas del plan de estudios y la provisión de recursos son fundamentales para esta apuesta inclusiva que abarca los diferentes niveles de educación y ofertas educativas.

Finalmente, vale la pena mencionar la intención política actual de combatir las desigualdades a la entrada y a la salida de la escuela. Este 
programa incluye la creación de un plan de no retención en educación básica (hasta el noveno grado). Este plan presupone un trabajo intensivo y diferenciado con las/los estudiantes que revelan las mayores dificultades (Governo de Portugal, s.f.).

Cabe señalar que, hace algunos años, solo el $60 \%$ de estudiantes que terminan el $12^{\circ}$ grado seguían estudiando, contando especialmente entre aquellos que no están en la educación formal, las y los jóvenes que han completado la formación profesional. Sin embargo, en ese año escolar hubo la mayor tasa de matrícula en educación superior (universidades y escuelas politécnicas), el mejor desde 2010, acercándose a los valores previos a la crisis. Medidas políticas como la extensión de la enseñanza obligatoria (2009), que ganó la efectividad en 2012/2013, Ilevando a que un mayor número de estudiantes tenían las condiciones para proceder a su registro; el crecimiento positivo de los resultados de los exámenes nacionales en las escuelas secundarias, en particular en 2015; y el aumento de confianza de los hogares sobre la economía después de cuatro años de crisis y de la intervención externa, son factores relevantes para este aumento de las entradas en la educación superior.

Hay también un vínculo profundo entre las opciones políticas nacionales y las directrices europeas para la educación. La necesidad de acercarse al modelo europeo ha estado en las raíces de la reforma educativa. La preocupación por la mejora de las cualificaciones dentro de la "sociedad del conocimiento" ha sido una racionalidad fundamental. Por ejemplo, la extensión a 12 años de escolaridad obligatoria, ya en 2009, se basó en la idea de que el país necesita una educación extendida para desarrollar la economía y posicionar a los individuos en el nuevo mercado de trabajo (Justino, 2010; Rodrigues, 2010). El aumento de las cualificaciones y la finalización de la educación secundaria vinieron en conjunción con la ampliación de la democratización y como un camino hacia la empleabilidad de las y los jóvenes (Silva, 2012).

Particularmente desde el comienzo de este milenio, las directrices de la UE enfatizan el crecimiento económico sostenido con más y mejores empleos y una mayor cohesión social; la promoción de la equidad y de la ciudadanía activa para responder a las necesidades de la economía basada en el conocimiento; y la preparación de las y los jóvenes para participar en una sociedad y una economía del conocimiento altamente competitiva. La educación y la formación se consideran factores clave del desarrollo socioeconómico. El riesgo de marginación, pobreza y 
exclusión económica y social de la población de ESL puede poner en riesgo todo el desideratum de la UE que se desprende de la Estrategia de Lisboa (EP, 2000) como la región más competitiva del mundo, como objetivos de la Estrategia Europa 2020. La reducción de la población de ESL, al mismo tiempo que se asegura las calificaciones y certificaciones a un mayor número de jóvenes, se considera esencial para el pleno empleo y la cohesión social.

En Portugal, la relación de las diversas partes interesadas (stakeholders) con las drivers y las rationales europeas es principalmente un esfuerzo de recontextualización: por un lado, para impulsar políticas dirigidas a la integración europea y, por otro, para implementar las políticas nacionales a través de políticas de renacionalización (Musselin y Paradeise, 2009). Esto se aplica a la relación entre la educación y el trabajo, entendida como un compromiso conjunto entre las partes interesadas. A menudo, los entrevistados en el proyecto RESL.eu hacen hincapié en una visión del déficit portugués en comparación con la UE y los países de la OCDE.

En el marco de la estrategia portuguesa en educación, formación y cualificación, se puede ver la influencia de la política de la UE en la lucha contra el abandono escolar prematuro en tres rationals principales: a) inclusión social; b) calidad y eficacia educativas; y c) calificaciones para el trabajo y el mercado laboral. Estas rationals asumen diferentes formas en las diferentes fases, que se mencionan arriba, y las expresan conjuntos particulares de medidas.

\section{Medidas intramuros para enfrentar el abandono escolar prematuro en Escuelas Secundarias Portuguesas}

En lugar de enfocar el ESL solamente como un problema social e individual que Portugal está encontrando difícil de manejar, una de las actividades del proyecto RESL.eu era una evaluación de las intervenciones intramuros (dentro de la escuela) y extramuros (fuera de la escuela) para reducir el abandono escolar prematuro en escuelas y otras entidades seleccionadas en el área de investigación de Porto. La investigación se ha llevado a cabo en 2014/2015 y destaca la concienciación local y la participación de diversas organizaciones educativas en el tratamiento de esta situación, mediante el desarrollo de medidas más o menos innovadoras. 
El trabajo de campo ha identificado un conjunto de medidas de diversa índole, muy articuladas con la naturaleza de cada organización y sus objetivos para los jóvenes.

A continuación, procedemos a la identificación de algunas de estas medidas, detectadas en un número de escuelas y que ilustran algo del trabajo contra el abandono escolar prematuro que se está haciendo en Portugal. Se procede despues a un análisis más cuidadoso de cuatro de estas medidas, desarrolladas en dos escuelas, que permiten una comprensión más amplia del trabajo empírico realizado por el proyecto RESL.eu.

En el plano del profesorado, se destacan medidas como la reflexión periódica y momentos de compartir entre profesores y la reorganización de los/as maestros/as de los grados 11 y 12 para proporcionar apoyo adicional a las/os estudiantes.

Al nivel del plan de estudios y de la práctica en el aula, se destaca la implementación de un tiempo extra con el asesor de educación de la clase; de un modelo pedagógico basado en la diferenciación y en el aprendizaje cooperativo; las horas de crédito en materias clave como Matemáticas y Portugués; la integración en diferentes clases para atender temas dejados atrás; la inversión en deportes bien como lo laboratorio de matemáticas y el club de ciencias.

En cuanto al apoyo para cada estudiante se destaca la inversión en la acción social escolar, la orientación psicológica y profesional y los servicios de integración profesional y social centrados en la promoción de los logros educativo; la acción tutorial de los docentes en horas flexibles; la aprendizaje y apoyo actitudinal a través de la oficina de apoyo al Estudiante y de la oficina para la mediación de conflictos y el apoyo a las necesidades educativas especiales. La implementación del Consejo de Delegados de Clase y de la Unión de Estudiantes se inserta en el nivel de participación ciudadana en la vida escolar.

En cuanto a la relación con la comunidad y la familia se cuentan medidas como la colaboración entre la escuela y las entidades asociadas (comunidad, empresas e instituciones públicas) para mejorar la transición al mercado de trabajo, las asociaciones con las familias y el desarrollo de la escuela para padres/madres con el fin de ayudarles en diversas habilidades de estudio, hábitos dietéticos y educación sexual, entre otros. 


\section{Un enfoque en una de las escuelas}

Se seleccionaron los casos de una escuela secundaria superior, que en el período de estudio desarrollaron medidas que se relacionan directamente con el abandono escolar prematuro: escuela B. El director abordó el proyecto pedagógico y la filosofía de la escuela en general y varias medidas que se llevaban a cabo en su escuela, con la preocupación por el rendimiento escolar y la reducción del abandono escolar temprano. Fue a partir de estas entrevistas que se seleccionaron las medidas a analizar. Se organizaron primero los grupos focales con las y los maestros de la escuela y más tarde con estudiantes, seguidos de entrevistas individuales con estudiantes seleccionados de los grupos.

\section{Una escuela en un área de intervención educativa prioritaria - Escuela B}

La escuela B pertenece a un grupo escolar, desde el pre primaria hasta el $12^{\circ}$ año y se encuentra en un área de intervención educativa prioritaria cubierta por el programa nacional TEIP, lo que significa que el absentismo escolar y el abandono escolar prematuro son aspectos centrales de la política escolar (para mayor discusión, véase Abrantes, Roldão, Amaral, y Mauritti, 2013). Al igual que varias y varios profesores, un informe oficial de la Inspeção Geral de Educação e Ciência (IGEC) se refirió al mantenimiento de altas tasas de absentismo escolar, abandono escolar prematuro, retención de grado y exclusión de estudiantes por ausencias injustificadas, especialmente en el nivel secundario.

La mayor parte de la población tiene un nivel socio económico bajo, situaciones de trabajo precario, economía paralela de supervivencia, y / o dependencia de los ingresos de inserción social. El porcentaje de los padres y madres de las y los estudiantes de la escuela secundaria con la educación superior es del 2\% (IGEC, 2013). De acuerdo con un informe oficial, el contexto social, económico y cultural de esta escuela no es favorable. (IGEC, 2013) o que pudiera influir en los bajos niveles de motivación de las y los estudiantes y mantiene el fracaso escolar.

En el año 2013/2014, la escuela tenía 200 estudiantes en la educación secundaria (cursos científicos y humanísticos), así como en el curso tecnológico y en la formación profesional (IGEC, 2013). En la educación secundaria, la escuela proporciona cursos científicos de Ciencia y Tecnología, Humanidades y Lenguas y cursos profesionales técnicos: 
Electrónica, Automatización y Computación; electricidad; turismo; restaurante; Apoyo a la Gestión del Deporte y Manutención de los niños.

El grupo de escuelas tenía más de 2000 alumnos de pre primario hasta el final de secundaria. El 99\% del alumnado del grupo de escuelas tenía la nacionalidad portuguesa. La mayoría de ellos eran "blancos" y hay algunos estudiantes de origen rumaní. En 2013-2014, el 53\% de estudiantes de la escuela secundaria recibieron el apoyo financiero de la ayuda económica de SASE (acción de apoyo social de la escuela). Más de la mitad de los estudiantes de la escuela secundaria tienen computadoras e Internet en el hogar (IGEC, 2013).

La escuela tiene una visión estratégica para apoyar el rendimiento escolar y mejorar la relación escuela-familia-comunidad con el fin de prevenir el abandono escolar prematuro, el absentismo escolar y la indisciplina. Esta estrategia se centra principalmente en dar más apoyo a la enseñanza y en el desarrollo de procesos de auto-aprendizaje. "Salir de la escuela sin calificación ha sido una preocupación" (Director).

Las principales medidas son preventivas, como se indica a continuación: La preparación para los exámenes, el defensor de las/os estudiantes, la provisión de tutorías: en la educación secundaria, el apoyo educativo se realiza en un espacio de autoaprendizaje con la supervisión del profesorado, la inversión en Deportes (como la gimnasia acrobática) y la cerámica; proyectos artísticos, como la Orchestra Orff, que apunta a la exploración de la música para alentar al alumnado del tercer ciclo de educación básica a mejorar su aprendizaje; o el proyecto piloto 10×10 en colaboración con el Teatro Nacional de São João ${ }^{6}$. Los artistas vienen a trabajar en el aula, en el nivel secundario. La bailarina que es experta en matemáticas y el director que sea actor y experto en portugués vienen a la escuela para motivar a las/os estudiantes con diferentes estrategias.

Estos recursos son el resultado de una fuerte red entre el profesorado, directores de clase, tutores, servicios técnicos y pedagógicos y padres y madres que es promovida por el blog de la escuela, el periódico de la escuela y su página web. Se explicarán dos de estas medidas.

\section{Preparación para los exámenes}

Hay cursos de preparación para los exámenes, que abarcan todos los ciclos, incluyendo el $12^{\circ}$ grado. Cada profesor tiene 45 o 90 minutos

6 Este proyecto está financiado por la Fundación Calouste Gulbenkian. 
semanalmente. Fue totalmente patrocinado por el Ministerio. Según el director "se utilizan estrategias diversificadas de enseñanza y aprendizaje según las dificultades experimentadas por los estudiantes".

El director es muy consciente de esta medida:

Sabiendo que algunos padres y madres no tienen posición económica y financiera, tenemos una medida, que se llama 'espacio de preparación para los exámenes.' Ha sido la palanca clave para la mejora de los resultados. Los estudiantes tienen apoyo sistemático durante los años escolares. Pueden siempre depender de ello sin gastar un centavo (director de la escuela).

La preparación del examen se centra en temas específicos que son objeto de exámenes nacionales. Se hace obligatorio a través de acuerdo entre la escuela y los padres/madres y se proporciona de forma gratuita:

No podemos obligar a los estudiantes a asistir cuando esto no es parte de su currículo como una asignatura obligatoria. El estudiante toma un documento en el que se informa al padre o tutor de que tiene este apoyo a Matemática, Portugués, Física y Química, etc. en un día dado en un momento dado. Si lo acepta, asume, a partir de ese momento, un carácter obligatorio (director de la escuela).

Según el director, la preparación para los exámenes hace que el alumnado se sientan más apoyados en su aprendizaje y fomenta el éxito escolar como una buena estrategia para mantenerlos en la escuela. La provisión de la preparación gratis del examen también impide que los padres/madres paguen para el apoyo privado adicional fuera de la escuela y para desmitificar que las y los profesores externos proporcionan servicios maejores que los internos:

La medida es supervisada por un coordinador y evaluada. Tenemos datos que nos permiten analizar el grado en que se está implementando esa medida, el número de estudiantes que asisten. Hay un coordinador para esa área (director de la escuela).

Las y los profesores, a su vez, son muy conscientes de esta medida, como lo demuestra la cantidad de atención que se le ha prestado durante lo grupo de discusión focal: 
Helena Costa Araújo, Eunice Macedo, António Magalhães y Cristina Rocha

Lo que tratamos de hacer con la preparación para los exámenes es estar a disposición de los estudiantes, apoyarlos para que puedan tener mejores resultados... No todas las disciplinas aún lograron las mejoras deseadas, pero lo harán con el tiempo. Tratamos de atraer a los estudiantes a estos apoyos que van más allá de las horas escolares, y motivarlos en la participación (maestra 12).

Su enfoque en la motivación del estudiantado y, en la oportunidad de desempeñarse mejor permite inferir que el problema es visto como estado de la motivación del estudiante y su bajo rendimiento. La preparación de exámenes es más dirigida a estudiantes del $12^{\circ}$ año en cursos científicos, pero también puede albergar a estudiantes en cursos profesionales que deseen tomar los exámenes para ir a la universidad. Los docentes reconocen la validez de la medida, pero algunos dudan o no reconocen su propósito de mantener a los estudiantes en la escuela.

En otro orden de ideas, otras u otros profesores reconocen el éxito de la medida y confían en su futuro:

Es todavía demasiado prematuro para hacer la evaluación, pero puedo decir que, por ejemplo, en términos de resultados de los exámenes, esta clase ha hecho mejor de lo que teníamos hace 3 años, con un grupo en el que había 15 estudiantes que fueron a la universidad (maestra 23).

La participación de las y los profesores es a veces como voluntarios que aceptan renunciar a su componente no docente convirtiéndolo en preparación para el examen.

Por último, las y los estudiantes son conscientes de la preparación del examen y la forma en que funciona. En algunos casos no relacionan la medida y el abandono escolar prematuro. No hay acuerdo sobre su carácter obligatorio después de involucrarse:

Yo he tenido apoyo de examen de geografía en el $11^{\circ}$ año. Estaba marcado ausente si no fui. Y luego, desde la tercera ausencia... Podría ser expulsado (estudiante 4).

Sin la medida yo mismo y otros saldremos de la escuela. El apoyo no es obligatorio. Si no asisten..., no tienen que dejar la escuela (estudiante 11). 
Como los exámenes nacionales cubren dos o tres años de los contenidos del curso, ayuda a los estudiantes a ponerse al día sobre los contenidos anteriores, dándoles mayores oportunidades de seguir a la educación superior. La familiaridad con los ejercicios de examen que permitirá un mayor éxito y la oportunidad de aprender de una manera constante es lo que está en juego para muchos estudiantes:

Es lo mismo para lo que hacemos en el aula... ejercicios similares pero diferentes. En este caso es bueno porque si es nuestro profesor quien está dando apoyo a los exámenes, él sabe dónde la clase tiene más dificultades y trabaja más duro allí. Las cosas se explican de una manera diferente, es más tranquilo; El maestro es más paciente (estudiante 20).

La necesidad de proporcionar conocimientos y apoyo emocional se expresan como buenas razones para participar:

Cuando me vi en medio de esos libros ni siquiera sabía por dónde empezar. Tener una persona que sabe, un maestro, creo que es bueno tener a alguien que nos apoya, no será tan difícil como pensábamos. Vamos a llegar allí y "puedo hacerlo" y nos dan apoyo tanto en el estudio como moralmente que "no será tan malo como creemos que lo hará" (estudiante 8).

Se puede decir que, en particular, con la extensión de la educación obligatoria (en 2009), la mayoría de los maestros ven la salida de la escuela sin una calificación como asunto de preocupación. Estas y estos profesores asumen su papel en tratar siempre de "arreglar algo que proteja de la deserción escolar", incluso cuando los factores externos pueden contrarrestar y limitar su acción. El riesgo de abandono o de interrupción temprana, aunque no muy significativo, en su escuela, es más notable en el primer período del $10^{\circ}$ año, cuando los estudiantes se enfrentan "por las primeras pruebas y empiezan a equiparar otras pistas, en particular, la ensenanza profesional". Estrategias tales como hacer que los estudiantes estudien en la escuela y disminuir el número de estudiantes por clase son medidas consideradas cruciales para promover el éxito y reducir el ESL.

La mayoría de los estudiantes se sienten bien apoyados por la escuela y sus maestros. Sin embargo, algunas luchas sociales fueron identificadas por parte de los estudiantes. Esto incluye los enfrentamientos en- 
tre estudiantes "blancos" y romaníes dentro de la escuela, la presencia conjunta de estudiantes de edades muy diferentes (10 a 20) y la falta de instalaciones adecuadas.

\section{Defensor del Estudiante}

El defensor de los estudiantes es un proyecto pionero dirigido a hacer que los estudiantes hablen de sus problemas (de aprendizaje, emocionales, de comportamiento). Su propósito es poner a los estudiantes de mayor edad a trabajar con los recién llegados bajo la supervisión de los docentes. Este es un bueno apoyo con el fin de tener mejores resultados y resolver los problemas cotidianos. Dentro de la oficina del defensor de los estudiantes, estos tienen la oportunidad de tomar decisiones y comprometerse en la planificación de actividades. El equipo del defensor de los estudiantes trabaja con la asociación de padres/madres.

La conciencia del diretor acerca del defensor de los estudiantes aparece visible en la descripción concreta y detallada y en su razonamiento:

Los jóvenes están familiarizados con esta medida a través del mensaje de todos los directores de curso, que son informados al comienzo del año escolar y tienen que transmitir este mensaje. Los padres/madres y tutores son informados en la reunión conmigo desde el principio del quinto grado; los estudiantes que son defensores hacen una visita a la escuela y sus instalaciones con los padres/madres y los nuevos estudiantes... Tengo dos, tres profesores que tienen horas de la componente no docente para este proyecto del defensor (director de la escuela).

La razón de participación del director parece estar relacionada con la voluntad de responder a las necesidades de la población escolar. Él tiene un fuerte sentido de la propiedad de esta y otras iniciativas:

Me gusta mucho ese proyecto. Algunos proyectos son casi mis hijos queridos (director de la escuela).

El director tiene una opinión clara sobre el alcance y el objetivo de la medida:

El propósito del defensor estudiantil es poner a los estudiantes mayores trabajando con los recién llegados, obviamente a través de los profesores, para entender cuáles son sus problemas, desde el punto de vista del aprendizaje 
y del comportamiento. Es decir, funciona como un ejercicio de ciudadanía activa y cotidiana, aplicándose luego a proyectos... (director de la escuela).

La falta de conciencia del profesorado sobre la medida se hace evidente en su pobre reflexión sobre ella durante las entrevistas y la discusión en grupo de enfoque. Cuando se requiere hablar sobre esta medida, las/os maestros cambian la conversación a otras materias. Esto puede ser ilustrativo de su falta de interés en la medida.

La mayoría de los estudiantes en el grupo nunca escucharon acerca de la medida, pero uno refiere Yo he oído hablar de ella en mi $9^{\circ}$ o $6^{\circ}$ grado... Es para ayudar a los estudiantes con dificultades... (estudiante 13). No se aclaran las razones de la participación. Un estudiante refirió que él nunca quiso ser un defensor, pero él sabía alguien que había sido.

Otro estudiante afirmó su desacuerdo con esta medida, ya que duda de la precisión de los estudiantes en la respuesta a las preguntas de otros estudiantes:

Pero ya dije que no estoy de acuerdo con el defensor del estudiante. Soy un buen estudiante de biología, pero también tengo dificultades. Hay cosas que no entiendo y otras cosas que entiendo, pero no sé cómo explicarlo. Creo que es incorrecto porque explicamos una manera, entonces el prof explica una manera diferente (estudiante 7).

Otro ve beneficios para sí mismo y para el otro en el uso de la medida:

No me importaría ayudar. Si sé que soy bueno en algo, no me importa ayudar a los demás. ¿Qué diferencia haría? Incluso es bueno para mí, eso me haría estudiar también (estudiante 19).

La buena intención del defensor de los estudiantes fue bien "anunciada" por el director, pero la falta de respuesta de otros interesados (stakeholders) mostró que no es muy reconocido dentro del grupo escolar.

\section{Para finalizar}

Se puede decir que la lucha contra ESL se encuentra en la tensión entre las políticas destinadas a hacer frente a las necesidades de la economía y de la sociedad del conocimiento y la tentativa de promover la inclusión 
Helena Costa Araújo, Eunice Macedo, António Magalhães y Cristina Rocha

social a través de la consecución de los itinerarios educativos, dando herramientas a las personas para llevar adelante sus posibles opciones.

El estudio ha puesto de manifiesto la estrecha relación entre las políticas europeas y las políticas nacionales. El éxito de la lucha contra el ESL puede ser relacionado con las políticas europeas -que eran importantes para llevar el tema a la agenda política nacional- y las políticas desarrolladas por el gobierno portugués. El pequeño número de medidas presentadas resulta meramente ilustrativo de los esfuerzos del gobierno portugués, de las escuelas y de sus actores, para producir una mayor inclusión de las y los jóvenes en la escuela y contribuir a la mejora de sus calificaciones. Si la primera es centrada en el aprendizaje de los contenidos disciplinarios y no hace más que reproducir el modelo escolar en un tiempo fuera de la escuela, la segunda se enfoca en apoyar el bienestar de las y los estudiantes, reconociendo este factor como condicionante de su aprendizaje. Estas y otras medidas que no ha cabido aquí mencionar, pero que contribuyen a la implicación de las y los jóvenes en la educación, pueden proporcionar acceso a educación superior y/ o una inserción menos frágil en el mercado laboral. Mucho más habría que decir acerca de las transformacións a nivel central y local producidas en el interior del sistema.

También es bastante notable el carácter político de las medidas aplicadas y su relación con las opciones políticas de los partidos en el gobierno, de manera que las medidas aplicadas en las escuelas reflejan o refractan las expectativas y representaciones sobre la educación que se pueden identificar al nivel meso y el nivel macro. Se hace referencia a algunas medidas, de cuatro de los contextos de estudio, que dan una breve descripción del trabajo desarrollado en Portugal en relación con ESL, pero no se mencionan muchas otras medidas desarrolladas dentro y fuera de las escuelas. Se hace hincapié en la fuerte hibridación del concepto de abandono escolar prematuro (ESL) y la diversidad de interpretaciones a las medidas en la lucha contra el ESL para las diferentes partes interesadas (directores/as, profesores/as y estudiantes) en función de las posiciones que ocupan en la jerarquía de la escuela.

Claramente, escuchar las diferentes voces de actores con diferentes posiciones dentro de la escuela permitió el acceso a su subjetividad interpretativa, lo que lleva a acentuar la necesidad de un mayor diálogo entre las partes. La producción de una cultura más coherente que uniera a las y los diferentes actores de la escuela podría estar en el horizonte 
Una mirada al abandono escolar prematuro en Portugal: realidades, contextos y prácticas

Helena Costa Araújo, Eunice Macedo, António Magalhães y Cristina Rocha

de este diálogo. Gran parte de las/os jóvenes valoran las medidas. Si nosotros podemos admitir las buenas intenciones inherentes a su creación e implementación, todavía parece haber espacio para que tengan un mayor impacto en la vida de ello, que parecen ser capaces de asumir y también reclamar una mayor centralidad dentro del sistema educativo.

\section{Referencias}

Abrantes, P., Roldão, C., Amaral, P., \& Mauritti, R. (2013). Born to fail? Some lessons from a national program to improve education in poor districts. International Studies in Sociology of Education, 23(1), 17-38. doi:10.1080/09620214.2013.770206

Araújo, H. C., Magalhães, A. M., Rocha, C., \& Macedo, E. (2014). Policies on early school leaving in nine European countries: A comparative analysis. Antwerp: UniversityofAntwerp.

Araújo, H. C., Macedo, E., Santos, S. A., \&Doroftei, A. O. (2015). Country report. WP3. Survey B: Case school descriptions. [Informe no publicado]. Porto: ClIEFPCEUP.

Borrás, S., \& Jacobsson, K. (2004). The open method of co-ordination and new governance patterns in the EU. Journal of European Public Policy, 11(2), 185-208. doi: 10.1080/1350176042000194395

Comisión Europea (2010). Comunicação da Comissão. Europa 2020. Estratégia para um crescimento inteligente, sustentável e inclusivo. Recuperado de http://eur-lex.europa. eu/LexUriServ/LexUriServ.do?uri=COM:2010:2020:FIN:PT:PDF

Conselho Nacional de Educação - CNE (2010). Estado da educação 2010. Percursos escolares. Lisboa: CNE.

Conselho Nacional de Educação - CNE (2011). Estado da educação 2011. A qualificação dos portugueses. Lisboa: CNE.

Conselho Nacional de Educação - CNE (2012). Estado da educação 2012. Autonomia e descentralização. Lisboa: CNE.

Dale, R. (2007). Globalization and the rescalling of educational governance. In C. A. Torres \& A. Teodoro (Eds.), Critique and utopia: New developments in the sociology of education in the twenty-first century (pp. 25-42). Plymouth: Rowman \& Littlefield Publishers.

Decreto de ley n. ${ }^{\circ}$ 54/2018, 6 de julho, Regime jurídico da educação inclusiva, Diário da República série I, n. ${ }^{\circ} 129$, p. 2918. Recuperado de https://dre.pt/application/conteudo/115652961

European Parliament - EP (2000). Lisbon European Council 23-24 March. Presidency conclusions. Recuperado de http://www.europarl.europa.eu/summits/lis1_en.htm

Eurostat (2019). Early leavers from education and training. Recuperado de https:// ec.europa.eu/eurostat/statistics-explained/index.php/Early_leavers_from_education_ and_training\#Overview

Governo de Portugal (s.f.). Programa do XXII Governo Constitucional 2019-2023. Recupe- 
rado de https://www.portugal.gov.pt/download-ficheiros/ficheiro.aspx?v=54f1146c05ee-4f3a-be5c-b10f524d8cec

http://www.oecd.org/edu/eag2013\%20(eng)--FINAL\%2020\%20June\%202013.pdf

Inspeção-Geral da Educação e Ciência - IGEC (2013). Relatório de atividades 2013. Lisboa: Inspeção-Geral da Educação e Ciência.

Justino, D. (2010). Difícil é educá-los. Lisboa: Fundação Francisco Manuel dos Santos.

Macedo, E., Araújo, H. C., Magalhães, A. M., \& Rocha, C. (2015). La construcción del abandono prematuro de la escuela como concepto político: Un análisis en la sociología de la educación. Profesorado: Revista de Curriculum y Formación del Profesorado, 19(3), 28-42.

Magalhães, A. M., Araújo, H. C., Macedo, E., \& Rocha, C. (2015). Early school leaving in Portugal: Policies and actors' interpretations. Educação, Sociedade \& Culturas, 45, 97-119.

Musselin, C., \& Paradeise, C. (2009). France: From incremental transitions to institutional change. In C. Paradeise, E. Reale, I. Bleiklie, \& E. Ferlie (Eds.), University governance: Western European comparative perspectives (pp. 23-49). Dordrecht: Springer.

Organización para la Cooperación y el Desarrollo Económicos, (2013). OECD Indicators. Recuperado de https://www.oecd.org/els/health-systems/Health-at-a-Glance-2013. pdf

Organización para la Cooperación y el Desarrollo Económicos, (2018). Education at a glance 2018. Recuperado de https://www.oecd-ilibrary.org/education/education-ata-glance-2018_eag-2018-en

Orden n. ${ }^{\circ}$ 6173/2016, 10 de maio, Estratégia de Educação para a Cidadania. Diário da República, 2. ${ }^{a}$ série - N. ${ }^{\circ}$ 90, p. 14676. Recuperado de https://www.dge.mec. pt/sites/default/files/Projetos_Curriculares/Aprendizagens_Essenciais/2016_despacho6173.pdf

Orden n. ${ }^{\circ}$ 5908/2017, 5 de julho, Projeto de autonomia e flexibilidade curricular dos ensinos básico e secundário, no ano escolar de 2017 -2018. Diário da República, 2. ${ }^{2}$ série - N. ${ }^{\circ} 128$, p. 13881. Recuperado de https://www.dge.mec.pt/sites/default/files/ Curriculo/Projeto_Autonomia_e_Flexibilidade/despacho_5908_2017.pdf

Rodrigues, M. L. (2010). A escola pública pode fazer a diferença. Coimbra: Almedina.

Silva, A. S. (2012). Políticas de educação para tornar efectiva a escolaridade obrigatória. In M. L. Rodrigues \& P. Adão e Silva (Eds.), Políticas públicas em Portugal (pp. 371379). Lisboa: Imprensa Nacional /ISCTE.

Stoleroff, A., \& Pereira, I. (2009). A reforma da carreira docente e a conflitualidade profissional: Análise da mobilização dos professores e de discursos sindicais. In P. Abrantes (Coord.), Actas do Encontro SocEd2009. Contextos Educativos na Sociedade Contemporânea (pp. 164-187). Lisboa: ISCTE.

Tarabini, A. y Montes, A., (2015). La agenda política contra el abandono escolar prematuro en España: La LOMCE contra las evidencias Internacionales. Avances in Supervisión Educativa, 23, 1-20.

UCAÇÃO - text 
